\title{
EVALUATION OF NUCLEAR CHANGES IN THE BUCCAL EPITHELIAL CELLS OF TOBACCO USERS IN NNEWI, SOUTH EAST NIGERIA
}

\author{
SAMUEL IFEDIORANMA OGENYI ${ }^{1 *}$, ANTHONY AJULUCHUKWUNGOKERE ${ }^{1}$, JONATHAN MADUKWE ${ }^{2}$
}

${ }^{1}$ Department of Medical Laboratory Science, Nnamdi Azikiwe University, Nnewi Campus, Nigeria. ${ }^{2}$ Department of Histopathology, National Hospital, Abuja, Nigeria. Email: si.ogenyi@unizik.edu.ng

Received: 12 June 2019, Revised and Accepted: 13 July 2019

\begin{abstract}
Objective: The objective of this study was to evaluate nuclear changes in the buccal epithelial cells of commercial bus drivers using tobacco in Nnewi. Tobacco use constitutes a major risk factor of oral lesions, due to its genotoxic and mutagenic effects on the buccal cavity epithelial cells.

Methods: One hundred and twenty-three subjects, consisting of 83 tobacco users (test group) and 40 tobacco and alcohol non-users (control group) were recruited for this cross-sectional study. Buccal smear was obtained from each participant, processed, and stained with the Papanicolaou staining method. Two hundred cells were counted for each stained slide and the percentage of various nuclear changes determined, and the repair index calculated. Comparisons of nuclear changes between and within groups were carried out using the Kruskal-Wallis $\mathrm{H}$-test with $\mathrm{p}<0.05 \mathrm{considered}$ significant.
\end{abstract}

Results: Micronuclei were the most prevalent nuclear changes (100\%) while broken egg nuclei were the least (15.2\%). Nuclear changes were most prevalent among snuffers $(33.42 \%)$. A statistically significant difference $(\mathrm{p}<0.05)$ was observed when the median values of nuclear changes were compared in different study groups and control subjects. Similarly, the prevalence of nuclear changes increased with the age of subjects and the duration of tobacco use, whereas the repair index decreased with increased prevalence.

Conclusion: Increased nuclear changes were observed in the buccal epithelial cells of tobacco users, with snuff inhalation slightly impacting more severe effects than cigarette smoking. Buccal smear cytology may constitute an effective screening method for precancerous lesions amongst tobacco users.

Keywords: Nuclear, Changes, Tobacco, Repair index

(C) 2019 The Authors. Published by Innovare Academic Sciences Pvt Ltd. This is an open access article under the CC BY license (http://creativecommons. org/licenses/by/4. 0/) DOI: http://dx.doi.org/10.22159/ajpcr.2019.v12i8.34551

\section{INTRODUCTION}

In Nigeria, tobacco is consumed mainly in two forms, smoked and smokeless tobacco products. Tobacco usage, in any form constitutes a major risk factor of oral cavity neoplasms $[1,2]$. Tobacco use plays a significant role in the prevalence of head and neck lesions [3]. Smoking is one of the greatest risk factors of lung cancers [4]. The carcinogenic effect of tobacco may be related to its genotoxic effects in the oral mucosal cell, where carcinogenesis may be initiated by the destructive effect of heat generated during smoking, the numerous chemical mutagens present in tobacco or by the interaction of chemical components of tobacco with the cells [1]. A dose- and time-dependent relationship exists between tobacco use and risk of development of oral lesions, with the form of tobacco use determining the degree of risk [5]. A study carried out by Biswas et al. [6] revealed that tobacco users have significantly higher oral cellular abnormalities when compared with healthy control subjects, with chewers of tobacco having worse cellular abnormalities than smokers. Prominent cellular abnormalities observed by these authors include condensed chromatin (CC), pyknosis, karyolysis, and bi nucleation, with the degree of atypia being age-dependent.

Kodiya et al. [7] observed that head and neck cancers (HNC) are one of the most common health problems in Nigeria, affecting relatively the youths, but due to paucity of literature on community-based studies to determine the incidence, the disease burden, pattern, and magnitude are obscured. Opubo et al. [8] reported 20-24 new cases in Northern Nigeria and $6.2 \%$ prevalence in Southern Nigeria. This corroborated an earlier report of 33-38 new cases by Amusa et al. [9]

Carcinogens and mutagens present in tobacco are capable of initiating genotoxic and nongenotoxic changes in the oral epithelial cells and a reduction in the level of blood antioxidant $[10,11]$. The damaged cells may progress to pre-neoplastic and neoplastic lesions by stimulating clonal proliferation [12]. Although the molecular changes may not be detectable by conventional histopathology, their accumulation over time, lead to massive individual cell nuclear changes which can be detected histologically or cytologically. The significance of bio-monitoring of nuclear abnormalities among tobacco users, using exfoliated cytology of oral mucosa cells, has been reported [13-15]. This study, therefore, becomes imperative, to unravel the pattern and extent of nuclear changes among tobacco users and to determine the repair index of the nuclear changes.

\section{MATERIALS AND METHODS}

Study area

This study was carried out in Nnewi Metropolis, Nnewi North Local Government Area Anambra State Nigeria

\section{Study population}

The study population comprised commercial bus drivers. One hundred and twenty-three subjects were recruited as stated bellow.

\section{Study design}

The study was a cross-sectional survey and the study groups were made up of the following:

- $\quad$ Cigarette smokers: $n=42$

- Snuff users: $n=41$

- Control: (no tobacco users) $n=40$.

\section{Ethical approval}

Ethical clearance to carry out this study was obtained from the Ethics Committee of the Faculty of Health Sciences and Technology, Nnamdi 
Azikiwe University, Nnewi Campus. Each participant read and signed a written, informed consent form.

\section{Sample collection}

Buccal smear was collected from the oral cavity of each participant with the aid of wooden spatula. This was preceded by the rinsing of the buccal cavity with table water to avoid contamination with debris. The smear was immediately emulsified in $10 \mathrm{ml}$ of $70 \%$ ethyl alcohol in a plastic universal container, labeled with identification numbers, and allowed to stand at room temperature for $12 \mathrm{~h}$, to complete fixation of cells.

\section{Sample processing}

The cell suspension in each universal container was mixed by shaking the container after which the content was emptied into a centrifuge tube. The samples were spun in a centrifuge for $5 \mathrm{~min}$ at $1200 \mathrm{rpm}$, the supernatant was discarded, the deposit re-suspended in the remaining fixative. A drop each was placed on pre-coated and pre-labeled slides and monolayer smears made with the aid of wire loop. The smears were allowed briefly to air dry and the slides stained by the Papanicolaou staining method.

\section{Microscopy and photomicrography}

All stained slides were examined under an optical microscope using $\times 10$ and $\times 40$ objective lenses. Two hundred cells were critically evaluated for cytoplasmic and nuclear details and the number of cells with various nuclear changes documented.

\section{Repair index}

The repair index for each sample was calculated by the formula described by Farhadi et al. [1]. It is the ratio of the sum of Karyorrhexis and Karyolysis and Broken nuclei and Micronuclei [RI= KR+KL/ $\mathrm{BE}+\mathrm{MN}]$ and measures the severity of nuclear damage and the fate of the dysplastic cell.

\section{Data analyses}

Numerical data were summarized using median, mean rank, and Chisquare, whereas categorical data were presented using frequency and percentages. Comparisons of nuclear changes across and within groups were determined using the Kruskal-Wallis H-test, $\mathrm{p}<0.05$ was considered significant. The associations of various forms of oral cellular nuclear changes with the repair index were determined using Spearman's correlation test. These statistical analyses were carried out with the aid of the Statistical Package for the Social Sciences (SPSS) version 22.0.

\section{RESULTS}

The results of the present study are presented in Tables 1-4 and Figs. 1-2 below.

Nuclear changes were more prevalent amongst snuff users (33.97\%) and least among control subjects (15.21\%) (Fig. 1).
Comparison of the median value occurrences of the various nuclear changes and repair index among cigarette smokers, snuffers, and control groups revealed statistically significant differences $(p<0.05)$. The mean rank values of pyknosis revealed a consistent decrease from cigarette smokers to snuffers and to control groups, whereas the repair index conversely showed a progressive increase in the same direction. Pairwise comparison of the nuclear changes and repair index between control and test groups showed increased statistically significant differences in pyknosis, karyolysis, micronuclei, and nuclear halo (Table 1).

There were statistically significant differences $(\mathrm{p}<0.05)$ in the median values of pyknosis, karyolysis, micronuclei and repair index when compared across the short-term, middle-term, and long-term duration (in years) of smoking among the cigarette smoker group. Conversely, the median values of karyorrhexis, karyomegaly, binucleate, broken egg nuclei, and perinuclear halo had no statistically significant differences $(p>0.05)$. There were increased statistically significant differences $(p<0.05)$ in the median values of pyknosis and micronuclei when compared between short-term and middle term and a decrease in middle term versus long-term comparison. Similarly, karyolysis and repair index increased significantly in a comparison between shortterm and middle term and middle term and long-term duration of smoking, respectively (Table 2).

Similarly, there were increased statistical significant differences $(p<0.05)$ in the median values of pyknosis, when compared between short-term and middle term, and a decrease in middle term versus long-term inhalation of tobacco comparison. Karyomegaly, on the other hand, increased significantly when compared between shortterm and middle term, short-term and long-term but decreased in the middle term versus long-term tobacco inhalation comparison. Median values of karyolysis, binucleate, and micronuclei similarly increased significantly when compared across the short-term and middle term, short-term and long-term, and middle term and long-term tobacco inhalation. The repair index values conversely decreased significantly when compared between short-term and middle term but increased in the short-term versus long-term and middle term versus long-term tobacco inhalation comparison. There was no statistically significant difference in karyorrhexis, broken egg nuclei, and nuclear halo when compared across the duration of tobacco inhalation (Table 3).

There were decreased statistically significant differences in the median values of pyknosis when compared between $35-40$ years and $>50$ years and $41-46$ years and $>50$ years. Karyolysis, on the other hand, increased significantly, when compared between 35-40 years and 41-45 years, $35-40$ years and $>50$ years, $41-46$ years and $>50$ and 46 years and $>50$ years of age. Karyomegaly decreased significantly when compared between 35-40 years and $41-45$ years, $35-40$ years and $>50$ years and $41-46$ years and $>50$ years and 46 years and $>50$ years of age. Binucleate, micronuclei, and repair index conversely increased significantly, when compared between 35-40 years and 41-45 years, 35-40 years and $>50$ years and 41-46 years and $>50$ years of age. The median value of

Table 1: Comparison of nuclear changes and repair index across control and test groups

\begin{tabular}{|c|c|c|c|c|c|c|}
\hline \multirow[t]{2}{*}{ Nuclear changes } & \multicolumn{2}{|c|}{ Control versus Smokers } & \multicolumn{2}{|c|}{ Control versus Snuffers } & \multicolumn{2}{|c|}{ Smokers versus Snuffers } \\
\hline & $\chi^{2}$ & p-value & $\chi^{2}$ & p-value & $x^{2}$ & p-value \\
\hline Pyknosis & $52.595^{*}$ & 0.000 & 7.689* & 0.006 & $63.220^{*}$ & 0.000 \\
\hline Karyorrhexis & 8.076 & 1.000 & $5.450^{*}$ & 0.020 & $5.199^{*}$ & 0.023 \\
\hline Karyolysis & $23.553 *$ & 0.000 & 2.261 & 0.133 & $4.504^{*}$ & 0.034 \\
\hline Karyomegaly & $30.444^{*}$ & 0.000 & $17.251^{*}$ & 0.000 & $37.357^{*}$ & 0.000 \\
\hline Bi-nucleate & 0.463 & 0.496 & $22.271^{*}$ & 0.000 & $29.975^{*}$ & 0.000 \\
\hline Broken egg nuclei & $9.628^{*}$ & 0.002 & 1.261 & 0.261 & $5.199^{*}$ & 0.023 \\
\hline Micronuclei & $59.911^{*}$ & 0.000 & 3.165 & 0.075 & $49.795^{*}$ & 0.000 \\
\hline Nuclear halo & $42.731 *$ & 0.000 & 1.261 & 0.261 & $63.220^{*}$ & 0.000 \\
\hline Repair index & $8.443^{*}$ & 0.004 & 1.517 & 0.218 & $13.743^{*}$ & 0.000 \\
\hline
\end{tabular}

*The Chi-square is significant $\mathrm{p}<0.05$ 
micronuclei, however, decreased significantly in a pairwise comparison between 41-46 years and $>50$ years of age (Table $4 a$ and $b$ ).

Photomicrographs of Papanicolaou stained buccal smears of the study subjects showing pyknosis, karyorrhexis, karyolysis, karyomegaly, binucleate, broken egg nuclei, micronuclei, and nuclear halo are shown in Fig. 2.

\section{DISCUSSION}

Buccal mucosal nuclear changes in subjects habitually and occupationally exposed to risk factors of oral lesions have been studied by a good number of researchers [1-14]. However, none seemed to have targeted the study population of the present study. The habitual use of tobacco amongst commercial bus drivers in Nigeria, though yet to be properly documented, is common knowledge. Mohan [16] earlier associated tobacco use; alcohol consumption and human papillomavirus infection with oral squamous cell carcinoma and according to him constitute the major risk factors of oral neoplasia.

The present study reported the highest percentage of participants within the age group of 50 years and above, and the majority of participants being smokers. Although most of the earlier studies paid little attention to age distribution, it could be noted here that majority of the participant is within the age group of 50 years and above is not unlikely related to the nature of the study population, who were mainly intra-state transports. Sequel to advancing in age, a good number of them preferred intra-state routes to the youth dominated inter-state routes. The report more so corroborated that of Khlifi et al. [2] who is a study of the cytogenetic abnormality in exfoliated buccal epithelial cells of HNC patients in a Tunisian population, reported that $60 \%$ of study subjects were $\geq 50$ years of age.

Pyknosis (P), karyorrhexis (KH), karyolysis (KL), karyomegaly (KM), binucleate (BN), broken egg nuclei (BE), micronuclei (MN), and nuclear

Table 2: Comparison of nuclear changes and repair index across the short-term, middle term and long-term cigarette smoking

\begin{tabular}{|c|c|c|c|c|c|c|}
\hline \multirow[t]{2}{*}{ Nuclear changes } & \multicolumn{2}{|c|}{ Short-term versus Middle term } & \multicolumn{2}{|c|}{ Short-term versus Long-term } & \multicolumn{2}{|c|}{ Middle term versus Long-term } \\
\hline & $\chi^{2}$ & p-value & $x^{2}$ & p-value & $\chi^{2}$ & p-value \\
\hline Pyknosis & 19.301* & 0.000 & 1.733 & 0.188 & $12.900^{*}$ & 0.000 \\
\hline Karyolysis & $5.282^{*}$ & 0.022 & 0.455 & 0.500 & 0.684 & 0.408 \\
\hline Micronuclei & $11.244^{*}$ & 0.001 & 2.056 & 1.000 & $9.327^{*}$ & 0.002 \\
\hline Repair index & 0.023 & 0.879 & 0.455 & 0.500 & $12.900^{*}$ & 0.000 \\
\hline
\end{tabular}

*The Chi-square is significant $\mathrm{p}<0.05$

Table 3: Comparison of nuclear changes and repair index across the short-term, middle term and long-term Snuff use

\begin{tabular}{|c|c|c|c|c|c|c|}
\hline \multirow[t]{2}{*}{ Nuclear changes } & \multicolumn{2}{|c|}{ Short-term versus Middle term } & \multicolumn{2}{|c|}{ Short-term versus Long-term } & \multicolumn{2}{|c|}{ Middle term versus Long-term } \\
\hline & $\chi^{2}$ & p-value & $\chi^{2}$ & p-value & $\chi^{2}$ & p-value \\
\hline Pyknosis & $18.000^{*}$ & 0.000 & 2.319 & 0.128 & $16.099 *$ & 0.000 \\
\hline Karyolysis & $18.000 *$ & 0.000 & $32.000 *$ & 0.000 & $32.000 *$ & 0.000 \\
\hline Karyomegaly & $18.000^{*}$ & 0.000 & $8.568^{*}$ & 0.003 & $4.174^{*}$ & 0.041 \\
\hline Bi-nucleate & $18.000 *$ & 0.000 & $4.900 *$ & 0.027 & $4.900^{*}$ & 0.027 \\
\hline Micronuclei & $18.000^{*}$ & 0.000 & $5.692 *$ & 0.017 & $5.692^{*}$ & 0.017 \\
\hline Repair index & $18.000 *$ & 0.000 & 16.099* & 0.000 & $16.099 *$ & 0.000 \\
\hline
\end{tabular}

*Significant level: $\mathrm{p}<0.05$

Table 4a: Comparison of the median values of nuclear changes and repair index across the various age groups of the study subjects

\begin{tabular}{|c|c|c|c|c|c|c|}
\hline \multirow[t]{2}{*}{ Nuclear changes } & \multicolumn{2}{|c|}{$35-40$ versus $41-45$} & \multicolumn{2}{|c|}{$35-40$ versus $46-50$} & \multicolumn{2}{|c|}{$35-40$ versus $>50$} \\
\hline & $\chi^{2}$ & p-value & $\chi^{2}$ & p-value & $\chi^{2}$ & p-value \\
\hline Pyknosis & 1.943 & 0.163 & 2.778 & 0.096 & $25.412^{*}$ & 0.000 \\
\hline Karyolysis & 3.173 & 0.075 & $29.000 *$ & 0.000 & $5.876^{*}$ & 0.015 \\
\hline Karyomegaly & $8.374 *$ & 0.004 & $6.473^{*}$ & 0.011 & 0.746 & 0.388 \\
\hline Bi-nucleate & $7.323^{*}$ & 0.007 & 0.077 & 0.782 & $19.765^{*}$ & 0.000 \\
\hline Broken egg nuclei & 3.173 & 0.075 & $14.246^{*}$ & 0.000 & 1.318 & 0.251 \\
\hline Micronuclei & $7.323^{*}$ & 0.007 & $14.246^{*}$ & 0.001 & $4.561^{*}$ & 0.033 \\
\hline Repair index & 8.993* & 0.003 & $29.000 *$ & 0.000 & 3.630 & 0.340 \\
\hline
\end{tabular}

*The Chi-square is significant $\mathrm{p}<0.05$

Table 4b: Comparison of the median values of nuclear changes and repair index across the various age groups of the study subjects

\begin{tabular}{|c|c|c|c|c|c|c|}
\hline \multirow[t]{2}{*}{ Nuclear changes } & \multicolumn{2}{|c|}{$41-45$ versus $46-50$} & \multicolumn{2}{|c|}{$41-45$ versus $>50$} & \multicolumn{2}{|c|}{$46-50$ versus $>50$} \\
\hline & $\chi^{2}$ & p-value & $\chi^{2}$ & p-value & $\chi^{2}$ & p-value \\
\hline Pyknosis & 0.163 & 0.686 & 18.179* & 0.000 & 0.914 & 0.339 \\
\hline Karyolysis & 7.778* & 0.005 & 0.007 & 0.932 & $8.647^{*}$ & 0.003 \\
\hline Karyomegaly & 7.778* & 0.005 & $6.631^{*}$ & 0.010 & $4.218^{*}$ & 0.040 \\
\hline Bi-nucleate & 7.778* & 0.005 & $4.731^{*}$ & 0.030 & $20.753^{*}$ & 0.000 \\
\hline Broken egg nuclei & 7.778* & 0.005 & 1.394 & 0.238 & $17.866^{*}$ & 0.000 \\
\hline Repair index & $13.686^{*}$ & 0.000 & $16.213^{*}$ & 0.000 & 0.506 & 0.447 \\
\hline
\end{tabular}




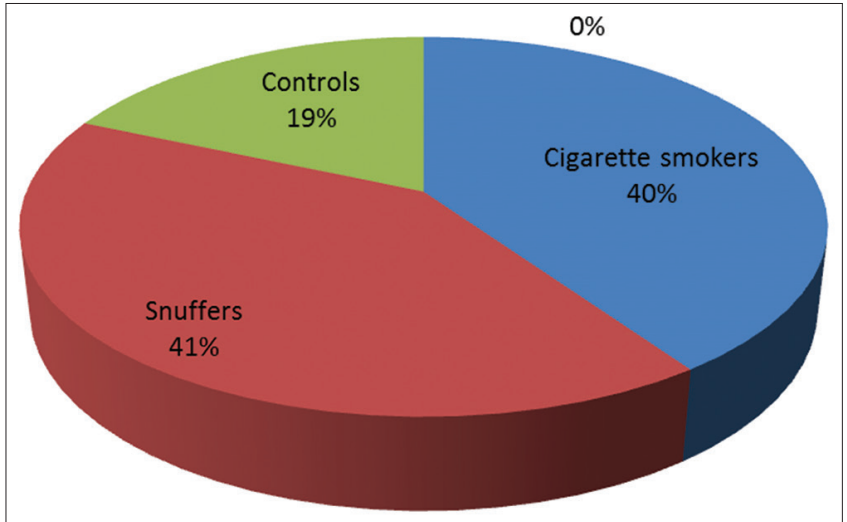

Fig. 1: Prevalence of nuclear changes among cigarette smoker, snuffers, and control groups $(n=123)$

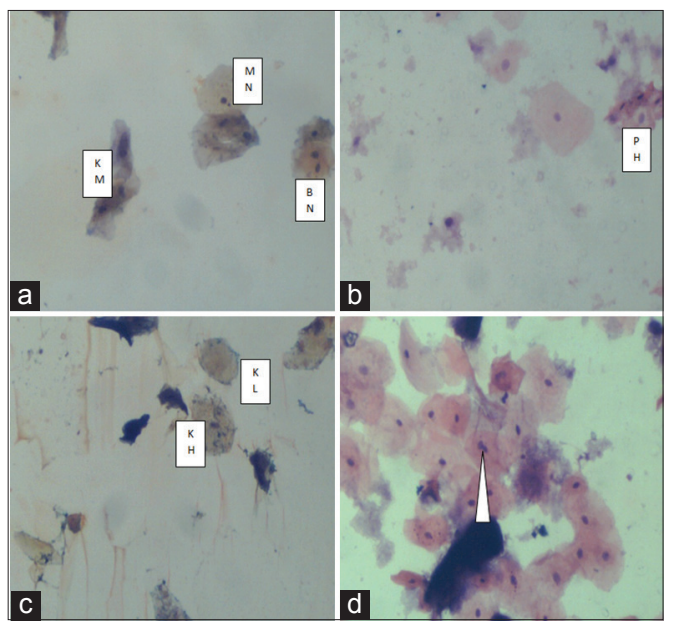

Fig. 2: Photomicrograph of buccal smear of test group showing; (a) micronucleus, karyomegaly, and bi-nucleate; (b) perinuclear halo; (c) karyolysis, and karyorrhexis; (d) broken egg nucleus $(\times 100$, PAP)

halo $(\mathrm{NH})$ were the nuclear changes reported in the oral mucosal cells of the subjects, with micronuclei being the most prevalent while snuffer group were the most affected. This report corroborates partly that of Farhadi et al. [13] who reported the higher occurrence of micronuclei in a study that involved smokers and non-smokers. Besides, the report of Farhadi et al., Khlifi et al. [2] observed a significantly elevated frequency of micronuclei and bi-nucleated cells in the buccal mucosa of HNC patients when compared with healthy controls. Khlifi et al. [2] also noted and reported a significant association of MN with tobacco smoking and chewing and alcohol drinking. Saranya and Sudha [14] in a similar study reported various cytomorphological changes in buccal mucosal epithelial cells of khaini (a product of tobacco) chewers in different age groups. These reports agreed with the findings of the present study.

Moreover, the nuclear changes herein reported corroborates with a much earlier study by Proia et al. [15] who reported that diverse buccal cellular changes are associated with smoking and smokeless tobacco. Biswas et al. [6] similarly observed and reported that CC, karyolysis $(\mathrm{KL})$, bi-nucleation, and pyknosis were the nuclear anomalies most frequently associated with tobacco smoking and chewing, thus strongly agreeing with the finding of the present study. Because of all these, it could be deduced that tobacco use induces varying nuclear changes in the oral mucosal cells of their consumers.

The high prevalence of nuclear changes among tobacco users (cigarette smokers and snuffers) though not directly reported in earlier studies, agree partly with the finding of Farhadi et al. [13] The authors who did not report the prevalence of these abnormalities among tobacco smokers reported very high occurrence of them amongst smokers when compared to non-smokers. The prevalence of nuclear changes, though highest among snuffers, was only very slightly higher than that of smokers. This finding agrees with the report of Khlifi et al. [2] who observed the higher occurrence of nuclear abnormalities among tobacco chewers than cigarette smokers. It could be explained by the fact that cigarette filters filter out some toxic agents of tobacco, thereby preventing direct contact with oral mucosa, which helps to reduce cellular damages. This is not the case with tobacco chewers and snuffers, whose oral/nasal mucosal cells are directly exposed to all the toxic substances of tobacco. Sequel to this, it could be inferred that both cigarette smoking and nasal inhalation of tobacco (snuffing) impact similar genotoxic effects on the oral mucosal cells. This study also reported a $100 \%$ prevalence of micronuclei, $97 \%$ of pyknosis, and a high prevalence of all other detected abnormalities. This agrees with earlier studies [1-14]. The relatively high prevalence of micronuclei in the control group (70\%) did not wholly agree with the earlier report of Farhadi et al. [13] and Khlifi et al. [2]. This finding though surprising is not unexplainable. This may be attributed to passive exposure to other agents of genotoxicity such as passive smoking, petrochemical products to mention but a few.

The present study, nonetheless, reported a significantly high prevalence of oral mucosal cell nuclear changes in the test subjects when compared with control subjects. This undoubtedly infers the genotoxic and/or carcinogenic effects of tobacco on the oral mucosal cells, thereby agreeing with Mohan [16] who reported tobacco use as the major risk factor of oral squamous cell carcinoma. It could also be inferred from the finding that though micronucleus assay is a wellvalidated method for testing genotoxic effects of various agents being the only biomarker that allows the simultaneous evaluation of both clastogenic and aneugenic effects in a wide range of cells, it should be used in conjunction with other biomarkers.

Furthermore, there were statistically significant differences when the medians values of nuclear changes and repair index were compared between control, a cigarette smoker and snuffer groups and this agrees with earlier reports $[2,13]$. These authors reported statistically significant differences in the mean distribution of pyknosis, karyolysis, karyorrhexis, bi-nucleate, broken egg nuclei, micronuclei, and repair index when compared in control and tobacco user group, which were also seen in this study. As aforenoted, these forms of nuclear abnormalities are validated biomarkers of cellular injury and genotoxicity and their significant increase in test subjects when compared to control is a clear indication that tobacco is an agent of genotoxicity. The higher value of the repair index in control and more so the increased the statistically significant difference in the median values, when compared with the test groups corroborates with the above assertion. This implies that though mild forms of nuclear changes (resulting maybe from inflammatory reactions) may be present in the buccal mucosa of non users of tobacco, the tendency of repair and reversal to normal cells is higher than in the dysplastic cells seen among tobacco users which more likely will progress from atypical to neoplastic cells, especially with continued use and the resultant continued cell damage. This could be better explained if one notes that the repair index, which is the ratio of the sum of karyorrhexis and karyolysis and broken egg nuclei and micronuclei [13], predicts the likelihood of neoplastic cellular transformation and invariably measures the degree of cellular damage. An inverse relationship, therefore, exists between the repair index and the likelihood of neoplastic cellular transformation. In light of this, therefore, it could be substantiated that tobacco-induced genotoxic effects on oral cells of the users.

The present study also reported a significantly increased in pyknosis, karyolysis, broken egg nuclei, and micronuclei among cigarette smokers when compared with snuffers. This may not conclusively infer more 
genotoxic and/or mutagenic effects of smoked tobacco compared with smokeless tobacco. This assertion could be held since karyorrhexis, karyomegaly, bi-nucleate, and perinuclear halo (other biomarkers of genotoxicity) were more prevalent amongst snuffers, who also had the highest overall number of nuclei anomalies. However, the increased statistically significant difference in the medians' values of pyknosis, karyomegaly, and bi-nucleate, when compared between cigarette smokers and snuffers, tend to support the fact that tobacco smoking slightly predisposes more risk to oral lesions than snuffing. This point could further be buttressed with the lower mean rank value of the repair index of smokers, though there was a non-significant difference in the distribution of their median values in a pairwise comparison within the groups.

\section{CONCLUSION}

It could be concluded from the findings of this present study that tobacco use is associated with nuclear changes, seen as nuclear abnormalities on the buccal mucosal cells of their users. Tobacco inhalation smoking (snuffing) impacts a slightly more severe effect than cigarette smoking. This was evident in the elevated prevalence of nuclear changes in snuffer and cigarette smoker groups compared to the non-tobacco control group.

\section{ACKNOWLEDGMENTS}

The authors wish to express their profound appreciation to the fleet managers of the garage where subjects were recruited for their cooperation.

\section{AUTHORS' CONTRIBUTIONS}

Samuel Ifedioranma Ogenyi designed the study, drafted the original manuscript, carried out the data analyses, and supervised the laboratory analyses.

Anthony Ajuluchukwu Ngokere reviewed the manuscript and participated in laboratory analyses.

Jonathan Madukwe carried out sample collection, sample processing, laboratory analyses, and also reviewed the manuscript.

All authors read and approved the final manuscript.

\section{COMPETING INTERESTS}

The authors declare that there are no conflicts of interest.

\section{REFERENCES}

1. Farhadi S, Jahanbani J, Jariani A, Ghasemi S. Bio-monitoring of the nuclear abnormalities in smokers using buccal exfoliated cytology. Adv Biores 2016;7:128-33

2. Khlifi R, Trabelsi-Ksibi F, Chakroun A, Rebai A, Hamza-Chaffai A. Cytogenetic abnormality in exfoliated cells of buccal mucosa in head and neck cancer patients in the Tunisian population: Impact of different exposure sources. Biomed Res Int 2013;2013:905252.

3. Rakesh KK, Shagufta P, Padma L, Samia R, Nisar AS. Prevalence and risk factors of gastroesophageal reflux disease (GERD) in adult Kashmiri population. Int J Pharm Pharm Sci 2018;10:6-62.

4. Muhas C, Kumar AV, Raja D. Etiological factors for the development of lung cancer in non-smokers: An overview. Int J Pharm Pharm Sci 2019;11:10-6

5. Haveric A, Haveric S, Ibrulj S. Micronuclei frequencies in peripheral blood and buccal exfoliated cells of young smokers and non-smokers. Toxicol Mech Methods 2010;20:260-6.

6. Biswas SD, Gara B, Banerjee M. Genotoxic effects of tobacco on buccal epithelium: Cell nuclear anomalies as biomarker. Asian Pac J Trop Med and Toxicol 2014;3:157-62.

7. Kodiya AM, Adamu AI, Nggada HA, Garandawa HI, Yusuf B. Ngamdu YB, et al. Epidemiology of head and neck cancers in Maiduguri-Northeastern Nigeria. Br J Med Med Res 2016;11:1-7.

8. Opubo BL, Abayomi OS, Wasiu LA. Current evidence on the burden of head and neck cancers in Nigeria. Head Neck Oncol 2009;1:1-14.

9. Amusa YB, Olabanji JK, Akinpelu VO, Olateju SO, Agbakwuru EA, Ndukwe N, et al. Pattern of head and neck malignant tumours in a Nigerian teaching hospital a ten year review. West Afr J Med 2004;23:280-5.

10. Rivera C. 4NQO carcinogenesis: A model of oral squamous cell carcinoma. Int J Morphol 2012;30:309-14.

11. Rivan VS, Bambang W, Merryana A, Sri S. The effects of exposure duration to electronic cigarette smoke on differences in superoxide dismutase and malondialdehyde in blood of wistar rats. Int J Curr Pharm Res 2019;11:13-6.

12. Miranda SR, Noguti J, Carvalho JG, Oshima CT, Ribeiro DA. Oxidative DNA damage is a preliminary step during rat tongue carcinogenesis induced by 4-nitroquinoline 1-oxide. J Mol Histol 2011;42:181-6.

13. Farhadi S, Sadri D, Sarshar S. Micronucleus assay of buccal mucosa: A useful noninvasive approach in screening of genotoxic nuclear damage. Adv Biores 2016;7:20-9.

14. Saranya RS, Sudha S. Cytomorpholo gical changes in buccal epithelial cells of khaini chewers in different age groups. Asian J Biomed Pharm Sci 2014;4:43-7.

15. Proia NK, Paszkiewicz GM, Nasca MA, Franke GE, Pauly JL. Smoking and smokeless tobacco-associated human buccal cell mutations and their association with oral cancer a review. Cancer Epidemiol Biomarkers Prev 2006;15:1061-77.

16. Mohan $\mathrm{H}$. The oral cavity and salivary glands. In: Textbook of Pathology. $6^{\text {th }}$ ed. New Delhi: Jaypee Brothers Medical Publishers; 2010. 